\title{
The Evolving Capabilities of Rhodopsin-based Genetically Encoded Voltage Indicators
}

Yiyang Gong*

\author{
Affiliations: \\ Department of Biomedical Engineering, Duke University, Durham NC 27708. \\ *Correspondence to: yiyang.gong@duke.edu
}

\begin{abstract}
Protein engineering over the past four years has made rhodopsin-based genetically encoded voltage indicators a leading candidate to achieve the task of reporting action potentials from a population of genetically targeted neurons in vivo. Rational design and large-scale screening efforts have steadily improved the dynamic range and kinetics of the rhodopsin voltage-sensing domain, and coupling these rhodopsins to bright fluorescent proteins has supported bright fluorescence readout of the large and rapid rhodopsin voltage response. The rhodopsin-fluorescent protein fusions have the highest achieved signal-to-noise ratios for detecting action potentials in neuronal cultures to date, and have successfully reported single spike events in vivo. Given the rapid pace of current development, the genetically encoded voltage indicator class is nearing the goal of robust spike imaging during live-animal behavioral experiments.
\end{abstract}

The desire for genetically encoded voltage indicators (GEVIs) that precisely read out the arbitrary spiking activity of multiple genetically targeted neurons stems from the large potential impact that these precise recordings of the spiking code can have at connecting neural activity to animal behavior $[1,2]$. Although genetically encoded calcium sensors offer similar targeting capabilities and continued development of these sensors has resulted in tools that can report the 
broad outlines of neural activity with high fidelity [3,4], GEVIs have the potential to read out the spike train with millisecond temporal resolution and map membrane potential hyperpolarizations, capabilities not present with calcium sensors. Voltage sensors developed over the past two decades have demonstrated improving signal-to-noise ratio (SNR) in well controlled culture experiments, but only moderate success when measuring action potentials in live-brain imaging preparations, where noise sources such as scattering, hemodynamics, and general background fluorescence wash out the small sensor response. Here we describe the progress of rhodopsin-based voltage sensors over the past four years, both in developing rhodopsins to serve as highly effective voltage-sensing domains (VSDs), and in developing methods to extract the information from these VSDs with high SNR. Fluorescent proteinrhodopsin fusion sensors represent the leading edge of voltage imaging tools when compared to the other members of the GEVI class, and are on the cusp of reporting neural spiking activity from live animal preparations.

Early generations of GEVIs fused voltage-sensitive phosphatases (VSPs) such as the Ciona intestinalis voltage-sensitive domain (Ci-VSD) with fluorescent proteins (FPs). In particular, the voltage sensitive fluorescent protein (VSFP) design attached pairs of FPs that interacted with fluorescence resonance energy transfer (FRET) to one Ci-VSD terminus [5-8] (Fig. 1a), or split the pair between the termini $[9,10]$. The fluorescence ratio of the two FPs thus reported the voltage-sensitive conformation of Ci-VSD with the high brightness of FPs. However, these sensors generally had slow voltage-sensing kinetics (>20 ms) and therefore had only small optical response to neuronal action potentials in the culture setting $(\sim 1 \% \Delta F / F)$. Subsequent engineering of the FP and VSD components resulted in sensors that reported the VSP conformation change with charge sensing FPs or allostery. In particular, Arclight (Fig. 1b) [11, 
12] and ASAP1 (Fig. 1c) [13] coupled a pHluorin mutant to Ci-VSD and a circularly permuted GFP to the homologous Galos galos VSD (gg-VSD), respectively, and currently represent the best engineered VSP-based sensors in terms of dynamic range (i.e. response to long voltage transients) and kinetics (i.e. response to short voltage transients) (Table 1).

Archaerhodopsin-3 (Arch) has simultaneously risen as an alternative VSD with voltagesensitive electronic configurations that modified the protein's absorption spectrum (Fig. 1d) [14]. The initial report of Arch voltage-sensitive fluorescence suggested that rhodopsins could serve as VSDs with fast and large dynamic range voltage response. Initial rational design then improved Arch using site-directed mutagenesis that drew heavily on existing literature detailing how mutations in the homologous bacteriorhodopsin might affect the rhodopsin photocycle [15-20]. These studies mutagenizing the charge centers of Arch that comprise the proton conduction pathway significantly impacted the kinetics and voltage sensitivity of the rhodopsin protonation event that supports voltage-sensitive absorption and fluorescence. Specifically, manipulation of the charge center D95 [21-24] eliminated the protein's native photocurrent, while manipulation of the charge center D106 [21, 22] increased the protein's voltage sensing kinetics. The rational designs improved the sensing dynamic range and kinetics of the Arch photocurrent-knockout mutants, but kinetics remained at $\sim 10 \mathrm{~ms}$, far slower than the kinetics of neural action potentials. In addition to rational approaches, more recent large-scale screening efforts using random mutagenesis and designed helix swapping led to the creation of QuasAr [25] and Archer [26], respectively. Both designs reduced voltage sensitive kinetics to $<1 \mathrm{~ms}$, and detected spikes with $\Delta F / F=25-50 \%$, while marginally increasing the sensor brightness (Table 1). As a trade-off for kinetics however, designs such as Archer had residual photocurrent because they did not employ the photocurrent reduction mutations in the proton conduction pathway [26]. In either case, the 
quantum yields of these sensors remained below $1 \%$, and high fidelity experimental recordings of spikes in culture settings required $\sim 1 \mathrm{~W} \mathrm{~mm}^{-2}$ excitation intensity. The dim fluorescence of these sensors, comparable in intensity to autofluorescence from bulk tissue, led to greatly reduced $\Delta F / F$ values during slice imaging experiments [25] and present further challenges for in vivo preparations such as phototoxicity or tissue heating.

Incidentally, FRET served as a mechanism to improve the effective quantum yields of rhodopsin VSDs while maintaining the fast, highly voltage-sensitive optical response. By fusing bright FPs, which served as the donor of the FRET pair and bright fluorescence readout, in close proximity to the rhodopsin ion channel, which served as the acceptor of the FRET pair and VSD, FP-rhodopsin sensors, or FRET-opsins, reported the voltage-sensitive rhodopsin electronic configuration and absorption with high brightness. Initial studies attaching fluorescent proteins to sensory rhodopsins [27] was able to extract the kinetics of various stages of the rhodopsin photocycle. Subsequently, two reports combined engineered rhodopsin VSDs such as the rhodopsin from L. Maculans (MacQ) [28] and QuasAr [29] with FPs in the FRET-opsin configuration. Although the Mac rhodopsin [30] originated from eukaryotes instead of archaea, its homology with Arch and bacteriorhodopsin enabled similar protein engineering to suppress the photocurrent and speed up the voltage sensing kinetics [28].

Successful design of FRET sensors hinges on maximizing FRET interaction by maximizing the spectral overlap and minimizing the physical distance between the donor and acceptor components, and these design parameters in FRET-opsin sensors were respectively limited by the availability of different colored FPs to couple to the rhodopsin absorption spectrum and the steric hindrance that prevents proper protein folding and membrane localization at short FP-rhodopsin distances. The FRET-opsin designs dealt with the former design parameter 
by using fluorescent proteins of various colors as the donor. Because the absorption spectra of Mac and QuasAr peaked similarly in the $\sim 560-580 \mathrm{~nm}$ range, yellow and orange FPs, such as mCitrine and mOrange2, maximized the spectral coupling and sensor $\Delta F / F[28,29]$. The FRETopsin designs dealt with the latter design parameter by modifying the linker length between the VSD and FP components. Reducing the linker length generally increased the FRET interaction and the $\Delta F / F$ response, but the sensor proteins failed to fold and localize to the neuron membrane at critically short linker lengths [28, 29].

The optimal Mac and QuasAr FRET-opsin sensors produced similar kinetics of $3 \mathrm{~ms}$ for the fast rise component that responded to voltage depolarizations and similar dynamic ranges, and thus produced similar $\Delta F / F \sim 5 \%$ in response to neuronal action potentials in culture (Fig. 2a, Table 1) $[28,29]$. Signal-to-noise ratio was defined as the ratio of the peak fluorescence transient response to the shot-noise limited baseline fluctuations, or SNR $=(\Delta F / F) \times \sqrt{N}$, with $N$ the number of photons collected per neuron per frame, and these culture experiments reached SNR $\geq 25$. The bright fluorescence of these sensors also enabled imaging of action potentials in intact slice tissue preparations, as the fluorescent protein read-out was significantly higher (510x) than the background autofluorescence, and had sufficiently low shot-noise limited background fluctuations to resolve individual action potentials from cortical pyramidal neurons with $\Delta F / F \sim 2.5 \%$ and SNR $>10$ (Fig. 2b) [28]. When tested in live animal preparations, MacQmCitrine reported the long-duration dendritic action potentials from cerebellar Purkinje neurons with $\Delta F / F \sim 1 \%$ and SNR $\sim 10$ (Fig. 2c), aided by the slow kinetics of these complex spikes that matched sensor kinetics and the large membrane surface area of the Purkinje neuron dendritic tree that elevated the effective brightness of the imaged neurons [28]. With these favorable 
conditions, MacQ-mCitrine was able to enter the regime of single spike detection in vivo, which was previously untrodden ground for the GEVI field.

The body of work using FRET-opsin sensors above highlights the inextricable relationship between the imaged cell type or preparation and the fidelity of voltage sensors. Even in controlled culture experiments, comparison of sensor performance in reporting action potentials is often confounded by the different experimental conditions such as optical excitation power, electrophysiology holding potentials and current injection protocols, or even culture conditions that lead to varied action potential waveforms. While benchmarking sensors directly in vivo using neurons with known physiology will be the ultimate test bed in determining sensor merit, concurrent reports of action potential shape and optical design with the corresponding SNR measures will greatly help in comparing various sensors on an equal footing.

After establishing these standards for culture-based assays, we previously described methods to compare sensor response to action potentials using both shot-noise limited SNR measures and the spike detection fidelity metric, $d$ ' [31], with both measures utilizing the experimentally measured $\Delta F / F$ and brightness of the sensors; whereas SNR described only the peak response of sensors relative to the shot-noise limited background, $d$ ' measured the spike detection fidelity by considering the full response waveform. FRET-opsin based sensors had superior brightness and $\Delta F / F$ response and thus led in spike detection metrics when compared to existing sensors (Fig. 3a).

The fast imaging frame rates required for catching the fast voltage fluorescence transients necessitated large values of SNR or $d^{\prime} \sim 10$ to accurately classify neuron spiking, which corresponds to approximately one false positive per hour of imaging at $1 \mathrm{kHz}$ frame rate while balancing the probability of false positives and false negatives. FRET-opsin sensors performed 
over this threshold in culture and slice settings for detecting pyramidal neuron spikes (Fig. 3a). However, experiments imaging inhibitory neurons expressing the same sensors in slice failed to meet this threshold, and suggest that imaging the similarly short action potentials of neocortical neurons in live-mice experiments, including non-stationary noise sources such as brain motion, blood flow, and enhanced scattering, is currently only on the cusp of realization. Given an estimate that sensors suffer an order of magnitude decrease in SNR when transitioning from culture experiments to live animal imaging due to the excess noise and shortened action potentials, $d^{\prime} \sim 100$ in culture is necessary to support in vivo imaging of spikes, and current FRET-opsin sensors are still factors of $\sim 3-4$ away.

The FRET-opsin sensors nevertheless typify the recent voltage sensors developments that have exponentially increased the capability to imaging spikes within fast-spiking trains, represented by the ratio of $d^{\prime}$ to the sensor response kinetics (Fig. $\mathbf{3 b}$ ). In order to continue the trend of increasing sensor performance, future development of FRET-opsin sensors will need to resolve how FRET impacts the 1) voltage sensitivity, 2) kinetics, and 3) spectral diversity of the sensors. First, optimized FRET sensors attain maximum sensitivity at the inflection point around $\sim 50 \%$ FRET efficiency, which translates to the FP channel having approximately half of the $\Delta F / F$ response of the rhodopsin channel. Current designs based on Mac and Quas Ar failed to attain this FRET efficiency even at short linker lengths, suggesting that superior spectral overlap between the FP donor emission spectrum and the rhodopsin acceptor absorption spectrum could improve sensor performance. Second, we observed that the FP fluorescence channel of the FRET-opsin sensors had slower kinetics than the kinetics observed when directly imaging the weak fluorescence of the rhodopsin VSDs (Table 1). This suggests that the broad spectral widths of fluorescent protein emission profiles may overlap with multiple portions of the rhodopsin 
absorption spectrum that undergo different kinetics during voltage sensing, and that better spectral engineering may improve sensor kinetics as well. Finally, as noted with previous chronic expression of rhodopsin-red fluorescent protein fusions in live animals, we observed intracellular aggregation when the orange version of the FRET-opsin sensors was expressed in mice [28], suggesting that the monomericity of some FP-rhodopsin fusions is insufficient to avoid large background. All three of these observations suggest that further development of the rhodopsin and FP components of the FRET-opsins sensors could improve their capabilities to meet the challenge of in vivo spike detection.

To conclude, the development of voltage sensors over the past few years have significantly improved the outlook on performing in vivo quantification of single spikes. The exponential growth in sensor performance must level off, due to intrinsic biophysical limitations of charge distribution between different rhodopsin electronic configurations or the physical constraints of the VSP conformation. However, due to the super-exponential reduction of spike detection error rates with increasing measures of SNR, even linear gains in sensor performance will lead to large gains in the ability to record spikes from neurons in live-animal preparations, or consequently, in the numbers of neurons simultaneously imaged. For example, a modest improvement from $d^{\prime}=2$ to $d^{\prime}=4$ decreases the spike detection error probability from $\sim 10^{-1}$ to $\sim 10^{-3}$. In our current imaging regime, out-of-plane fluorescence from non-specifically labeled neuronal processes contributes large background emission that lowers the SNR. Thus, combining GEVIs with appropriate imaging modalities that provide depth sectioning and genetic labeling strategies that target ever smaller pools of neurons relevant to animal behaviors will enhance the capabilities of GEVIs as well. The current rate of sensor advancement and potential assistance from optical and genetic engineering suggest that robust live-animal voltage imaging of action 
potentials is nearing manifestation. 


\section{Acknowledgements}

We gratefully acknowledge research funding from DARPA, an NIH Pioneer award, the National Academies Keck Futures Initiative (NAKFI) research grant, the Stanford BioX Interdisciplinary

Initiatives Program, and the Stanford $C N C$ program. We also thank Mark J. Schnitzer for comments on this manuscript. 


\section{Figure Captions}

Figure 1 | Multiple voltage sensor configurations report membrane voltage using different mechanisms

GEVIs in the past decade of development have used primarily VSPs or rhodopsins as VSDs. Multiple sensor designs have reported voltage-sensitive conformation or electronic configuration from VSDs.

(a) The VSFP configuration fused FP pairs that interact with FRET to VSPs. During voltagesensitive conformation changes, the donor (FP1) and acceptor (FP2) of the FRET pair the physically shift, leading to enhanced FRET interaction, decreased donor emission, and increased acceptor emission.

(b) Arclight fused a mutant of pHluorin to VSPs, and the FP readout decreased intensity with voltage depolarization.

(c) ASAP1 fused a circularly permuted GFP to the extracellular terminals of a VSP. The voltage sensitive conformation manipulated the GFP structure via allostery, and decreased the sensor fluorescence intensity with voltage depolarization.

(d) Inhibitory rhodopsin proteins derived voltage sensitivity from the Schiff base protonation site within the proton-conduction pathway. During voltage depolarization, protonation of the Schiff base increased, leading to increases in rhodopsin absorption and fluorescence emission at constant excitation power.

(e) FRET-opsin fusions with bright FPs serving as the FRET donor reported the voltage-sensitive absorption of the rhodopsin FRET acceptor. During voltage depolarization, rhodopsin absorption increased, and the FP fluorescence decreased. Because the FP fluorescence quantum yield was orders of magnitude higher than the rhodopsin fluorescence quantum yield, the FP channel had 
much higher shot-noise limited SNR than the rhodopsin channel, and optical experiments using FRET-opsin sensors imaged only the FP channel.

Figure 2 | FRET-opsin sensors are able report action potentials in culture, slice, and in vivo preparations

(a) Image of a cultured neuron transfected with MacQ-mCitrine (left) with the associated optical fluorescence trace (center, top) and corresponding simultaneous electrophysiology trace (center, bottom). Spike-triggered average fluorescence (right, top) and electrophysiology (right, bottom) traces, obtained with $n>20$ spikes are also shown.

(b) Image of a cortical pyramidal neuron in a slice mouse brain preparation transfected with MacQ-mCitrine using in utero electroporation (left), and the associated optical fluorescence and electrophysiology traces in the same format as panel a (center and right).

(c) Image of a Purkinje cerebellar neuron (left, red dashed highlight) virally transduced with MacQ-mCirtrine from an optical-window live mouse imaging experiment. The associated fluorescence trace (center) shows fluorescence transients at similar rates and amplitudes as dendritic cerebellar Purkinje cell spikes in mice. Spike-triggered average fluorescence response (right) with $n \sim 10$ spikes is also shown.

Scale bars for images are $20 \mu \mathrm{m}$.

Images and data reproduced from ref. [28].

Figure 3 | FRET-opsin sensors outperform the current generation of voltage sensors in SNR when reporting single action potentials 
(a) Characterization of voltage sensors yielded experimental quantification of sensor $\Delta F / F$ response, brightness, and optical response waveforms. The combination of these metrics resulted in the plotted data points. The isocontours (dashed lines) show sensors of equal performance by the $d^{\prime}$ 'metric, which considered the full optical waveform response [31], or by the SNR metric, which was the ratio of the peak optical $\Delta F / F$ response to the shot-noise limited fluorescence background fluctuations. The FRET-opsin sensors are on the leading edge of sensor capabilities using either spike detection metric, and current culture and slice experiments using these sensors support high fidelity spike classification with $d^{\prime}>10$. Data reproduced from ref. [28].

(b) Sensor capabilities to detect action potentials within fast spiking trains in culture settings, i.e. the ratio of $d$ ' to the sensor response time constant $(\tau)$, have increased exponentially as a function of time over the past 3 years, again with FRET-opsin sensors at the head of this development. Given the pace of sensor development, the field is quickly nearing the threshold for performing live animal spike imaging experiments. 
Table 1 | Voltage sensor kinetics and spike detection metrics

\begin{tabular}{|c|c|c|c|c|c|c|c|c|c|c|}
\hline & \multicolumn{2}{|c|}{ + Kinetics } & \multicolumn{2}{|c|}{ - Kinetics } & $\begin{array}{c}\text { Experimental } \\
\text { Excitation } \\
\text { Wavelength }\end{array}$ & $\begin{array}{c}\Delta F / F \\
\text { (spike) }\end{array}$ & $\begin{array}{c}\text { Relative } \\
\text { Brightness }\end{array}$ & $d^{\prime}$ (spike) & $\begin{array}{c}\text { Bleaching } \\
\text { Rate }\end{array}$ & Reference \\
\hline Sensor & $\begin{array}{l}\tau_{\text {fast }} \\
(\mathbf{m s})\end{array}$ & $\begin{array}{l}P_{\text {fast }} \\
(\%)\end{array}$ & $\begin{array}{c}\tau_{\text {fast }} \\
(\mathbf{m s})\end{array}$ & $\begin{array}{l}P_{\text {fast }} \\
(\%)\end{array}$ & $(\mathrm{nm})$ & $(\%)$ & & & $\%$ per s & \\
\hline $\begin{array}{l}\text { Arclight- } \\
239\end{array}$ & 9 & 50 & 17 & 79 & 488 & 3 & 0.2 & 13 & 0.6 & [12] \\
\hline ASAP1 & 2.1 & 60 & 2.0 & 44 & 488 & 5 & 0.1 & 9 & 0.3 & [13] \\
\hline QuasAr & 0.3 & 62 & 0.3 & 73 & 640 & 48 & $<0.01$ & NR & NR & [25] \\
\hline Archer & $<1$ & NR & NR & NR & 655 & $25-40$ & $<0.01$ & NR & NR & [26] \\
\hline $\begin{array}{l}\text { MacQ- } \\
\text { mCitrine }\end{array}$ & 2.8 & 74 & 5.4 & 77 & 500 & 5 & 1.0 & 30 & 1.3 & [28] \\
\hline $\begin{array}{l}\text { QuasAr- } \\
\text { mCitrine }\end{array}$ & 3.1 & 62 & 4.8 & 38 & 488 & 5 & NR & NR & NR & [29] \\
\hline
\end{tabular}

The ' + ' and '-' kinetics modeled the voltage sensor's optical responses to depolarizing and hyperpolarizing voltage transients using bi-exponential fits to the experimental data, respectively. In brief, $P_{\text {fast }}$ is the percentage of the amplitude associated with the fast component in response to depolarizing and hyperpolarizing voltage transients, and $\tau_{\text {fast }}$ is the fast time constants of the response to depolarizing and hyperpolarizing voltage transients. NR - not reported. 


\section{References}

1. Peterka, D.S., Takahashi, H., and Yuste, R. (2011). Imaging voltage in neurons. Neuron 69, 9-21.

2. Mutoh, H., Akemann, W., and Knopfel, T. (2012). Genetically engineered fluorescent voltage reporters. ACS Chem Neurosci 3, 585-592.

3. Akerboom, J., Chen, T.W., Wardill, T.J., Tian, L., Marvin, J.S., Mutlu, S., Calderon, N.C., Esposti, F., Borghuis, B.G., Sun, X.R., et al. (2012). Optimization of a GCaMP calcium indicator for neural activity imaging. J Neurosci 32, 13819-13840.

4. Chen, T.W., Wardill, T.J., Sun, Y., Pulver, S.R., Renninger, S.L., Baohan, A., Schreiter, E.R., Kerr, R.A., Orger, M.B., Jayaraman, V., et al. (2013). Ultrasensitive fluorescent proteins for imaging neuronal activity. Nature 499, 295-300.

5. Lundby, A., Mutoh, H., Dimitrov, D., Akemann, W., and Knopfel, T. (2008). Engineering of a genetically encodable fluorescent voltage sensor exploiting fast Ci-VSP voltage-sensing movements. PLoS One 3, e2514.

6. Tsutsui, H., Karasawa, S., Okamura, Y., and Miyawaki, A. (2008). Improving membrane voltage measurements using FRET with new fluorescent proteins. Nat Methods 5, 683-685.

7. Lundby, A., Akemann, W., and Knopfel, T. (2010). Biophysical characterization of the fluorescent protein voltage probe VSFP2.3 based on the voltage-sensing domain of Ci-VSP. Eur Biophys J 39, 1625-1635.

8. Lam, A.J., St-Pierre, F., Gong, Y., Marshall, J.D., Cranfill, P.J., Baird, M.A., McKeown, M.R., Wiedenmann, J., Davidson, M.W., Schnitzer, M.J., et al. (2012). Improving FRET dynamic range with bright green and red fluorescent proteins. Nat Methods 9, 1005-1012.

9. Akemann, W., Mutoh, H., Perron, A., Park, Y.K., Iwamoto, Y., and Knopfel, T. (2012). Imaging neural circuit dynamics with a voltage-sensitive fluorescent protein. J Neurophysiol 108, 23232337.

10. Mishina, Y., Mutoh, H., Song, C., and Knöpfel, T. (2014). Exploration of genetically encoded voltage indicators based on a chimeric voltage sensing domain. Frontiers in Molecular Neuroscience 7.

11. Cao, G., Platisa, J., Pieribone, V.A., Raccuglia, D., Kunst, M., and Nitabach, M.N. (2013). Genetically targeted optical electrophysiology in intact neural circuits. Cell 154, 904-913.

12. Barnett, L., Platisa, J., Popovic, M., Pieribone, V.A., and Hughes, T. (2012). A fluorescent, genetically-encoded voltage probe capable of resolving action potentials. PLoS One 7, e43454.

13. St-Pierre, F., Marshall, J.D., Yang, Y., Gong, Y., Schnitzer, M.J., and Lin, M.Z. (2014). High-fidelity optical reporting of neuronal electrical activity with an ultrafast fluorescent voltage sensor. Nat Neurosci 17, 884-889.

14. Kralj, J.M., Douglass, A.D., Hochbaum, D.R., Maclaurin, D., and Cohen, A.E. (2012). Optical recording of action potentials in mammalian neurons using a microbial rhodopsin. Nat Methods 9, 90-95.

15. Lanyi, J.K. (1992). Proton transfer and energy coupling in the bacteriorhodopsin photocycle. J Bioenerg Biomembr 24, 169-179.

16. *Lanyi, J.K. (2004). Bacteriorhodopsin. Annu Rev Physiol 66, 665-688.

17. Kolodner, P., Lukashev, E.P., Ching, Y.C., and Rousseau, D.L. (1996). Electric-field-induced Schiffbase deprotonation in D85N mutant bacteriorhodopsin. Proceedings of the National Academy of Sciences of the United States of America 93, 11618-11621.

18. Marinetti, T., Subramaniam, S., Mogi, T., Marti, T., and Khorana, H.G. (1989). Replacement of aspartic residues $85,96,115$, or 212 affects the quantum yield and kinetics of proton release and uptake by bacteriorhodopsin. Proceedings of the National Academy of Sciences of the United States of America 86, 529-533. 
19. Moltke, S., Krebs, M.P., Mollaaghababa, R., Khorana, H.G., and Heyn, M.P. (1995). Intramolecular charge transfer in the bacteriorhodopsin mutants Asp85-->Asn and Asp212-->Asn: effects of pH and anions. Biophys J 69, 2074-2083.

20. Kalaidzidis, I.V., and Kaulen, A.D. (1997). Cl- -dependent photovoltage responses of bacteriorhodopsin: comparison of the D85T and D85S mutants and wild-type acid purple form. FEBS Lett 418, 239-242.

21. *Gong, Y., Li, J.Z., and Schnitzer, M.J. (2013). Enhanced Archaerhodopsin Fluorescent Protein Voltage Indicators. PLoS One 8, e66959.

22. *Maclaurin, D., Venkatachalam, V., Lee, H., and Cohen, A.E. (2013). Mechanism of voltagesensitive fluorescence in a microbial rhodopsin. Proceedings of the National Academy of Sciences of the United States of America 110, 5939-5944.

23. Park, J., Werley, C.A., Venkatachalam, V., Kralj, J.M., Dib-Hajj, S.D., Waxman, S.G., and Cohen, A.E. (2013). Screening fluorescent voltage indicators with spontaneously spiking HEK cells. PLoS One 8, e85221.

24. Venkatachalam, V., Brinks, D., Maclaurin, D., Hochbaum, D., Kralj, J., and Cohen, A.E. (2014). Flash memory: photochemical imprinting of neuronal action potentials onto a microbial rhodopsin. J Am Chem Soc 136, 2529-2537.

25. *Hochbaum, D.R., Zhao, Y., Farhi, S.L., Klapoetke, N., Werley, C.A., Kapoor, V., Zou, P., Kralj, J.M., Maclaurin, D., Smedemark-Margulies, N., et al. (2014). All-optical electrophysiology in mammalian neurons using engineered microbial rhodopsins. Nat Meth 11, 825-833.

26. *Flytzanis, N.C., Bedbrook, C.N., Chiu, H., Engqvist, M.K., Xiao, C., Chan, K.Y., Sternberg, P.W., Arnold, F.H., and Gradinaru, V. (2014). Archaerhodopsin variants with enhanced voltagesensitive fluorescence in mammalian and Caenorhabditis elegans neurons. Nat Commun 5, 4894.

27. Bayraktar, H., Fields, A.P., Kralj, J.M., Spudich, J.L., Rothschild, K.J., and Cohen, A.E. (2012). Ultrasensitive measurements of microbial rhodopsin photocycles using photochromic FRET. Photochem Photobiol 88, 90-97.

28. $\quad{ }^{* *}$ Gong, Y., Wagner, M.J., Zhong Li, J., and Schnitzer, M.J. (2014). Imaging neural spiking in brain tissue using FRET-opsin protein voltage sensors. Nat Commun 5, 3674.

29. **Zou, P., Zhao, Y., Douglass, A.D., Hochbaum, D.R., Brinks, D., Werley, C.A., Harrison, D.J., Campbell, R.E., and Cohen, A.E. (2014). Bright and fast multicoloured voltage reporters via electrochromic FRET. Nat Commun 5.

30. Waschuk, S.A., Bezerra, A.G., Jr., Shi, L., and Brown, L.S. (2005). Leptosphaeria rhodopsin: bacteriorhodopsin-like proton pump from a eukaryote. Proceedings of the National Academy of Sciences of the United States of America 102, 6879-6883.

31. *Wilt, B.A., Fitzgerald, J.E., and Schnitzer, M.J. (2013). Photon shot noise limits on optical detection of neuronal spikes and estimation of spike timing. Biophys J 104, 51-62. 


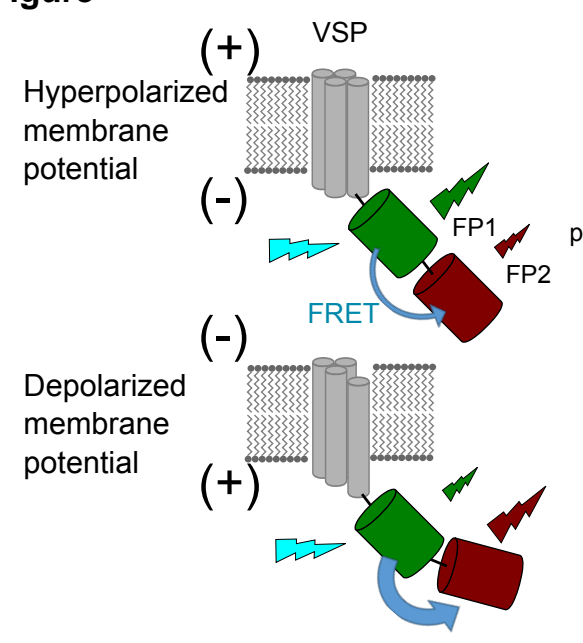

(a) VSFP
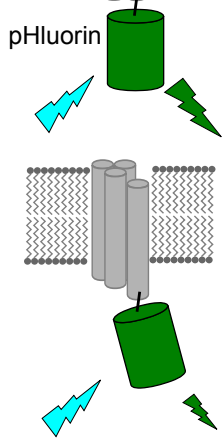

(b) Arclight

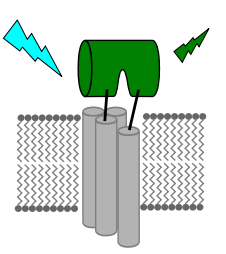
(c) ASAP1

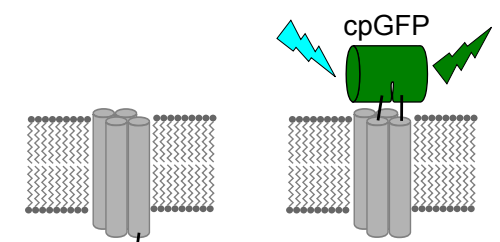

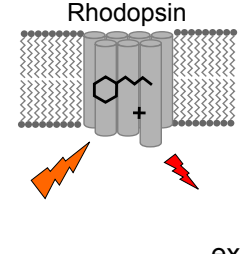
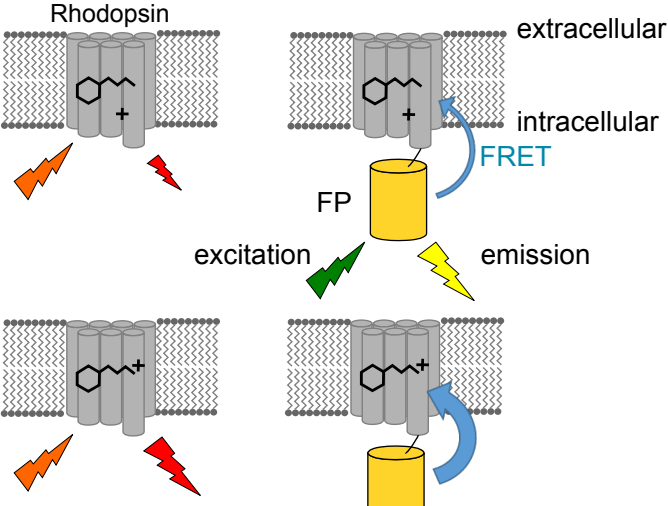

excitation

FP $\checkmark$ FRET

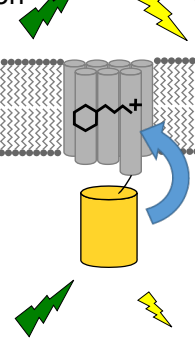

emission

Figure 1

(d) Rhodopsin

(e) FRET-opsin 
(a)

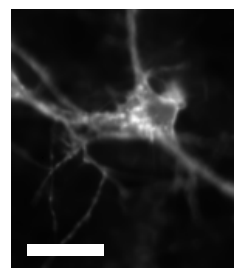

(b)

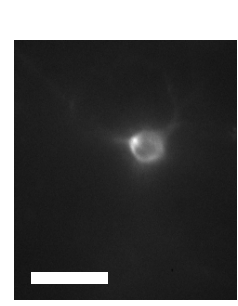

(c)

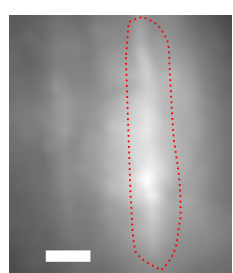

$\Delta F / F$
$-5 \%$

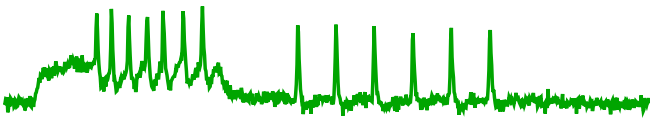

somy_Lalluth

Figure 2
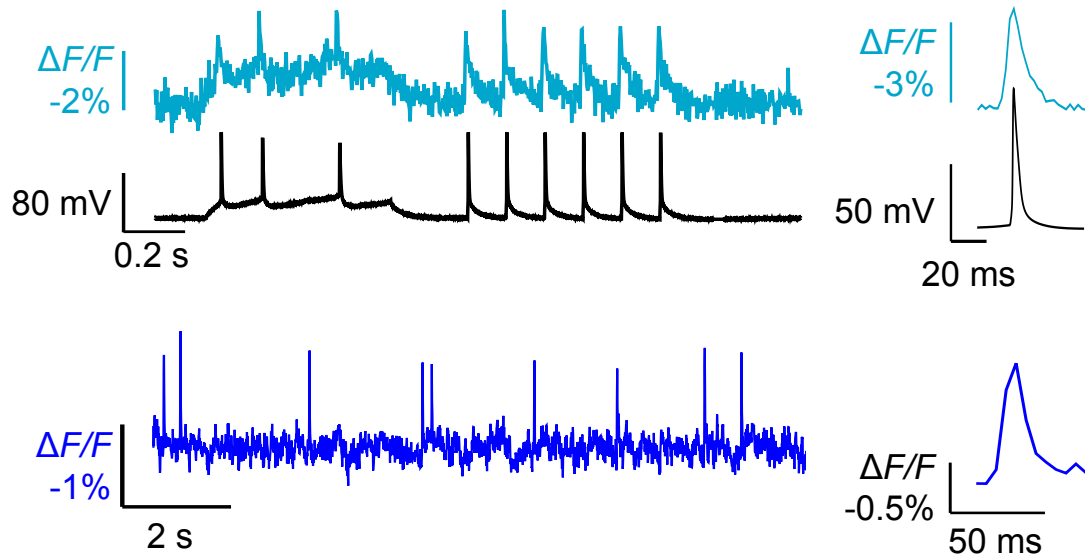


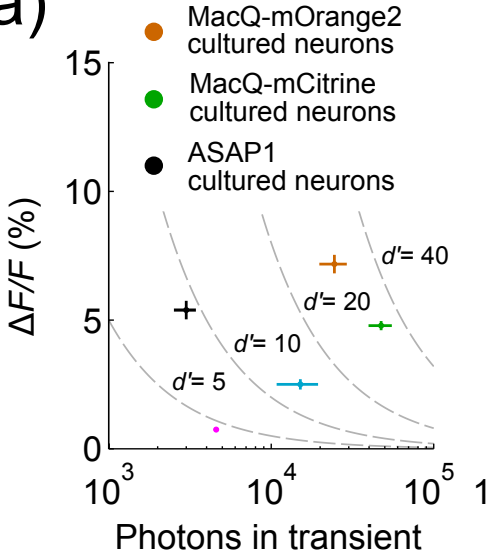

(b)

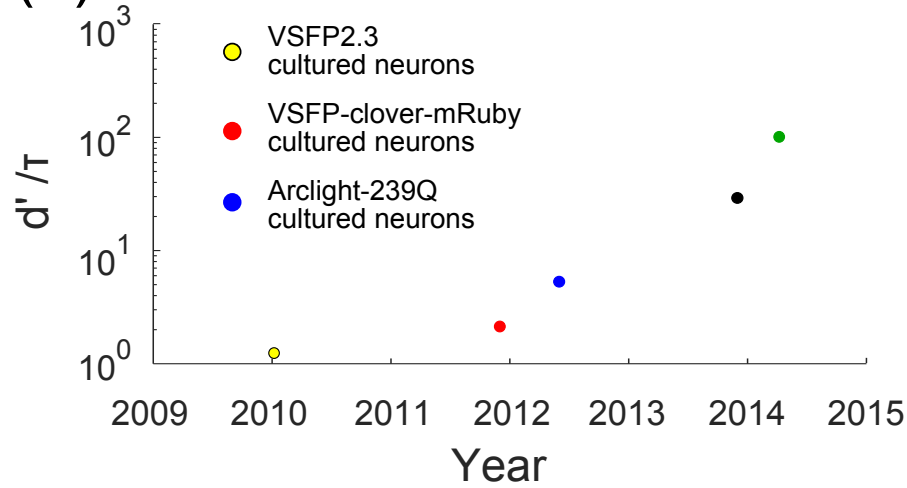

